\title{
Research on Multiparameters relation of Hyperlens
}

\author{
Junxian Ma \\ College of Information Engineering, \\ Shenzhen University, \\ Shenzhen, People's Republic of China \\ e-mail:majx@szu.edu.com \\ Xiaopeng Li \\ College of Information Engineering, \\ Shenzhen University, \\ Shenzhen, People's Republic of China
}

\author{
Qiulin Xiong \\ College of Information Engineering, \\ Shenzhen University, \\ Shenzhen, People's Republic of China
}

\author{
Wenjing Guo \\ College of Information Engineering, \\ Shenzhen University, \\ Shenzhen, People's Republic of China
}

\begin{abstract}
Optimization of hyperlens structure and composition of materials is a key problem for its application in biological imaging and nanometer lithography. This paper systematically optimizes and analyzes overall structure of the curved multilayer hyperlens. The influences of structure parameters, such as layer number, layer thickness, filling ratio, permittivities, curvature radius, thickness of photoresist (PR) and reflector layer (PRL) are numerically emulated. It shows that the full width at half maximum (FWHM), the contrast and the electric field intensity of the output beam can be comprehensively influenced by these parameters. As a result, $\mathrm{Ag}$ and $\mathrm{Al2O3}$ is the best metal and dielectric, less layer number and thinner layer thickness will be better, the optimal size of the curvature radius may fluctuate for practical fabrication of hyperlens, Ag is the best selection for PRL and $\mathrm{Cu}$ can sometimes be the substitute for decreasing the cost, The optimal PRL thickness varies from 40 to $80 \mathrm{~nm}$ and the optimal range of $P R$ thickness is between $30 \mathrm{~nm}$ and $50 \mathrm{~nm}$.

Keywords-metarmaterial;hyperlens;parameters optimazation; ; surface plasmon;FDTD solution
\end{abstract}

\section{INTRODUCTION}

Hyperlens, also called curved multilayer superlens, which can break through the diffraction limit, has received much extensive attentions in theoretical research and experimental production[1-3]. It is necessary to optimize the hyperlens, PRL, PR, and even mask's parameters for improving image features including resolution, contrast, and image intensity[4]. The existing simulation parameters includes layer number, layer thickness, filling ratio, wavelength, permittivity of metal and dielectric, curvature radius, PR thickness, PRL material and its thickness. Weiping Wang simulated and analyzed the impact of structure parameters of hyperlens on sub-diffraction imaging. He found the FWHM reach the minimum when the output interface radius value is $365 \mathrm{~nm}(\lambda)$, while the output intensity decreases and the beam divergence increases when the structure curvature radius increases from $\lambda / 2$ to $3 \lambda / 2$ [5]. Dong-dong Li optimized the performance of the hyperlens by adjusting geometric parameters. It showed that the performance of the hyperlens improves as the number of periods increases, but the improvement starts to saturate as the number of periods reaches a critical level[6]. Ji-Gang Hu found that the intensity and the FWHM of the output beam can be controlled by the structure parameters and operating wavelength. He adopted $365 \mathrm{~nm}$ as the optimized working wavelength to balance beam transmitted intensity and divergence of the beam, he also found that two factors work together to affect the output beam intensity and FWHM[7]. Heng-yi Li suggested the thickness of PR can't be too thin or too thick for the surface plasma excitation of PRL[8].

So imaging performance of the hyperlens cannot to be partially considered. In fact, the influence of structure parameters should be thought as a whole. Similarly, indexes that measure whether the parameters contribute to the resolution also mutually restrict each other. Based on the existing simulation researches, this paper aims to find the optimized parameters by coordinating the relation between parameters and materials in a systemic environment.

\section{SIMULATION AND RESULTS ANALYSIS}

The FDTD solution model of the curved multilayer hyperlens lithography system[9] is shown in Fig .1 and we are in the effective medium regime when $\mathrm{d} \leqslant \lambda \leqslant$ $\mathrm{r}_{\text {inner }}[10]$.

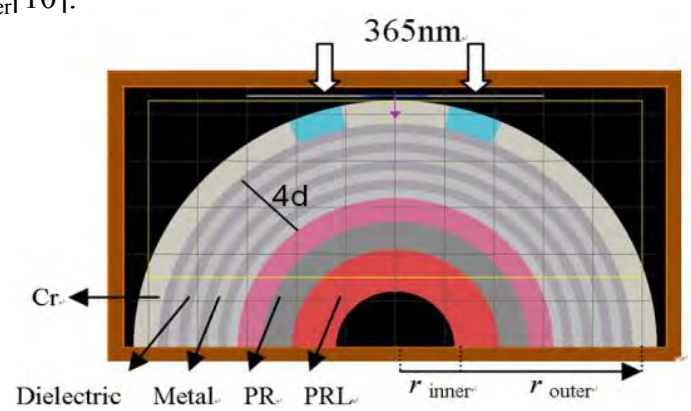

Figure 1. Simulation model of curved multilayer hyperlens lithography system. Wavelength of the incident TM polarization light is $365 \mathrm{~nm}$,

Chromium mask is fixed at $50 \mathrm{~nm}$ thick, the slit width in the mask is

$50 \mathrm{~nm}$ with $200 \mathrm{~nm}$ center-to-center space, Each unit of the periodic curved multilayer structure consists of one metal layer and one dielectric layer. 
The principle of curved multilayer hyperlens imaging can be expressed by dispersion equation of electromagnetic waves in cylindrical coordinates[11].

$$
k_{r}^{2} / \varepsilon_{\theta}-k_{\theta}^{2} /\left|\varepsilon_{r}\right|=\omega^{2} / c^{2}
$$

Where $\varepsilon_{\theta}$ and $\varepsilon_{r}$ are medium permittivities in transversal and radial direction respectively, $k_{\theta}$ and $k_{r}$ are corresponding wave vectors. Given proper matching permittivities of metal and dielectric, the transversal wave vectors $k_{\theta}$ gradually compressed along radial direction. It can be captured by conventional lens to form an image with sub-diffraction resolution.

Table I. ( A, B ) lists the initial parameters of our experiments, each column containing nine parameters presents an experiment. We change only one of them to obtain optimal parameters.

TABLE I. PARAMETERS OF THE EXPERIMENTS(A)

\begin{tabular}{|c|c|c|c|c|c|c|}
\hline Items & 1 & 2 & 3 & 4 & 5 & 6 \\
\hline Layer number & $4,6,10,14,18,22$ & 8 & 8 & 8 & 8 & 8 \\
\hline Layer thickness(nm) & 20 & $\begin{array}{r}8,10,15,20, \\
25,30,35,40\end{array}$ & 10 & 10 & 10 & $8,10,12$ \\
\hline Curvature radius(nm) & Inner: 320 & Inner :200 & Inner:220-820 & Inner :200 & Inner :200 & Inner :200 \\
\hline$\varepsilon_{\mathrm{m}}$ & $-2.40+0.25 i$ & $-2.40+0.25 \mathrm{i}$ & $-2.40+0.25 \mathrm{i}$ & $\begin{array}{c}-2.08+7.03 \mathrm{i} \\
-2.19+4.95 \mathrm{i},-2.40+0.25 \mathrm{i}\end{array}$ & $-2.40+0.25 \mathrm{i}$ & $-2.40+0.25 \mathrm{i}$ \\
\hline$\varepsilon_{\mathrm{d}}$ & 3.217 & 3.217 & 3.217 & 3.217 & $3.217,2.29,2.17$, & 3.217 \\
\hline Filling ratio & 0.5 & 0.5 & 0.5 & 0.5 & 0.5 & $0.4,0.5,0.6$ \\
\hline PRL & 0 & 0 & 0 & 0 & 0 & 0 \\
\hline PRL thickness(nm) & 0 & 0 & 0 & 0 & 0 & 0 \\
\hline PR thickness (nm) & 40 & 40 & 50 & 50 & 50 & 40 \\
\hline
\end{tabular}

TABLE II. PARAMETERS OF THE EXPERIMENTS (B)

\begin{tabular}{|c|c|c|}
\hline $\mathbf{7}$ & $\mathbf{8}$ & $\mathbf{9}$ \\
\hline 8 & 8 & 8 \\
\hline 10 & 10 & 10 \\
\hline Inner $: 320$ & Inner $: 320$ & Inner $: 320$ \\
\hline$-2.40+0.25 \mathrm{i}$ & $-2.40+0.25 \mathrm{i}$ & $-2.40+0.25 \mathrm{i}$ \\
\hline 3.217 & 3.217 & 3.217 \\
\hline 0.5 & 0.5 & 0.5 \\
\hline $\mathrm{Ag}, \mathrm{Cu}, \mathrm{Ni}, \mathrm{Fe}, \mathrm{Al}$ & $\mathrm{Ag}$ & $\mathrm{Ag}$ \\
\hline 50 & $20,40,60,80,100,120$ & 50 \\
\hline 50 & 50 & $20,40,60,80,100$ \\
\hline
\end{tabular}

\section{A. The impact of medium material}

Given same thickness of the medium $(p=0.5)$, permittivities in transversal and radial direction are calculated by:

$$
\varepsilon_{r}=\frac{2 \varepsilon_{m} \varepsilon_{d}}{\varepsilon_{m}+\varepsilon_{d}} \quad \varepsilon_{\theta}=\frac{\varepsilon_{m}+\varepsilon_{d}}{2}
$$

Here we assume $\mathrm{Al}_{2} \mathrm{O}_{3}$ as the ideal dielectric material and select $\mathrm{Ag}, \mathrm{Al}, \mathrm{Ni}$ as the candidates for optimal metals, Fig .2(a) shows three dispersion relation curves of the three metals. Obviously, Ag has the flattest curve among the three exciting metals, which means more transversal wave vectors are compressed along radial direction and the ray divergence is the minimal. So we fixed $\mathrm{Ag}$ to experiment the dielectric materials among $\mathrm{Al}_{2} \mathrm{O}_{3}, \mathrm{SiO}_{2}$ and PMMA. According to Fig .2(b), we believe PMMA is the optimal dielectric. In order to prove this idea we simulated electric field intensity distribution of the three parameters in Fig .3. Unexpectedly, the FWHM of electric field intensity of $\mathrm{Ag}-\mathrm{SiO}_{2}$ and $\mathrm{Ag}-\mathrm{PMMA}$ is $66 \mathrm{~nm}$, which is wider than that of the $\mathrm{Ag}-\mathrm{Al}_{2} \mathrm{O}_{3} 56 \mathrm{~nm}$. We can also find the difference from Fig .3 that the ray divergence of Ag$\mathrm{Al}_{2} \mathrm{O}_{3}$ is the smallest. Here the theory curves cannot match with the simulation result very well, which caused by light-admitting quality of the material $\mathrm{SiO}_{2}$ and PMMA.
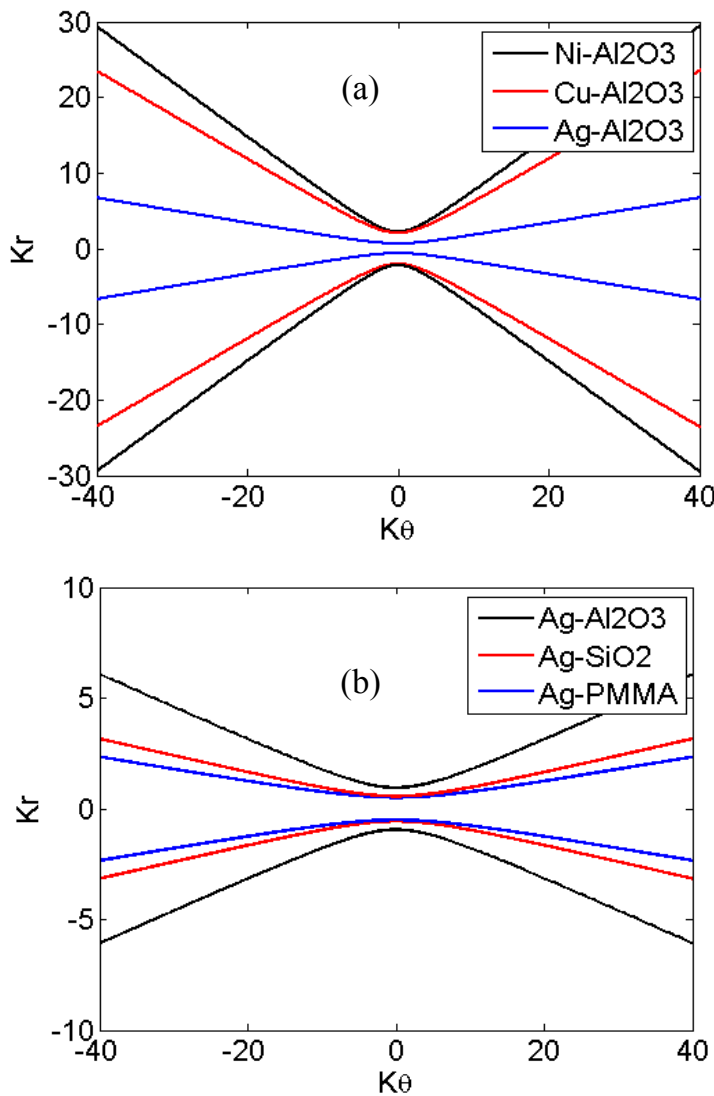

Figure 2. Dispersion relation curves of three metals(a) and three dielectrics (b). 


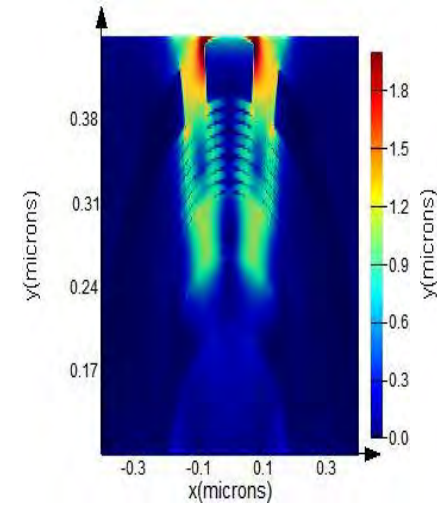

(a) Ag-PMMA

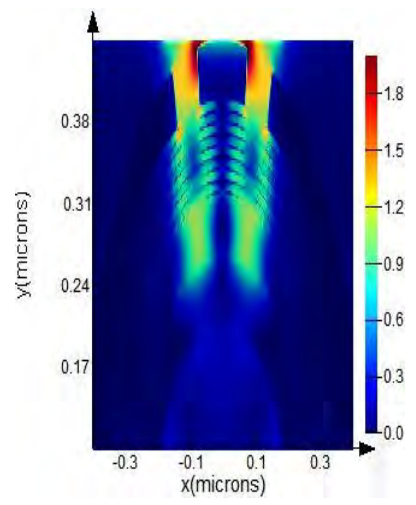

(c) $\mathrm{Ag}-\mathrm{SiO}_{2}$

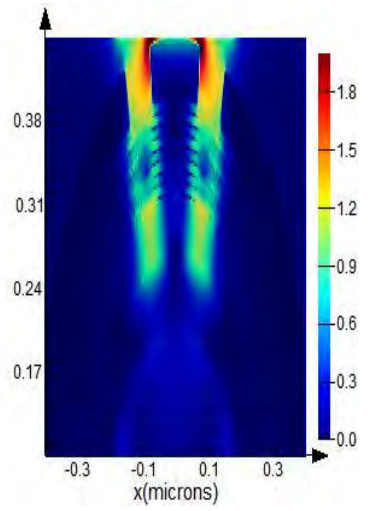

(b) $\mathrm{Ag}-\mathrm{Al}_{2} \mathrm{O}_{3}$

Figure 3. (a-c)The electric field intensity distribution of three different dielectrics

\section{B. The impact of geometric parameters}

The filling ratio is expressed by the equation $p=d_{m} /\left(d_{m}+d_{d}\right)$, where $d_{m}$ and $d_{d}$ are thickness of metal and dielectric respectively. We can expand the permittivities of the medium in transversal and radial direction by:

$$
\varepsilon_{\theta}=p \varepsilon_{m}+(1-p) \varepsilon_{d} \quad \varepsilon_{r}=\frac{\varepsilon_{m} \varepsilon_{d}}{p \varepsilon_{d}+(1-p) \varepsilon_{m}}
$$

We simulate filling ratio from 0 to 1 with a step size of 0.1 and select 0.4-0.6 to show the respective dispersion relation of the hyperlens, as shown in Fig .4. Obviously, the curve $p=0.5$ is the flattest, so we adopt $p=0.5$ as the optimal filling ratio based on the $\mathrm{Ag}-\mathrm{Al}_{2} \mathrm{O}_{3}$ structure. Of course, different structure may lead to different $p$.

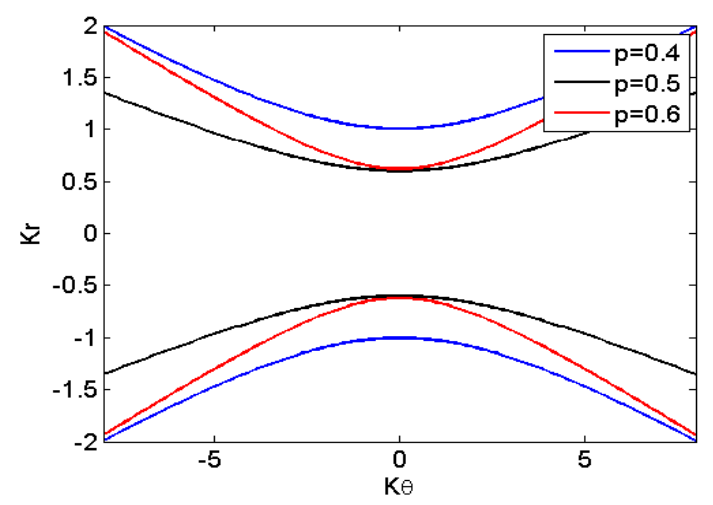

Figure 4. Dispersion relation curves of different filling ratio.

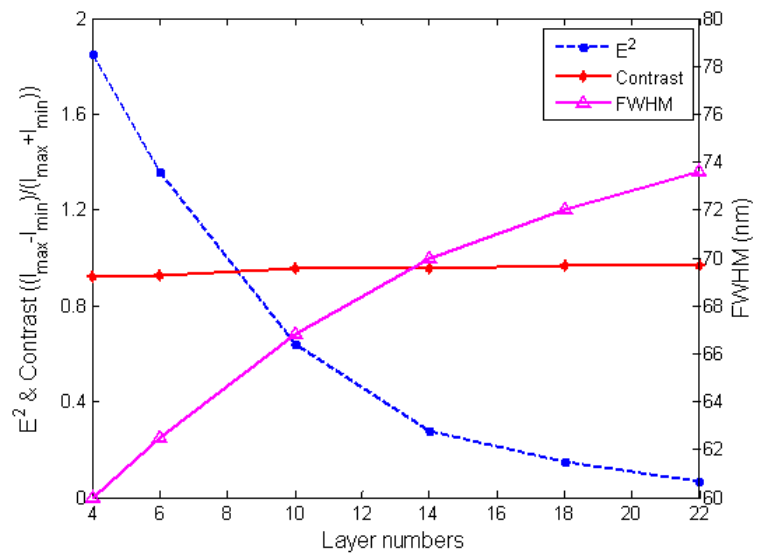

Figure 5. Relation curves between imaging indexes and layer numbers.

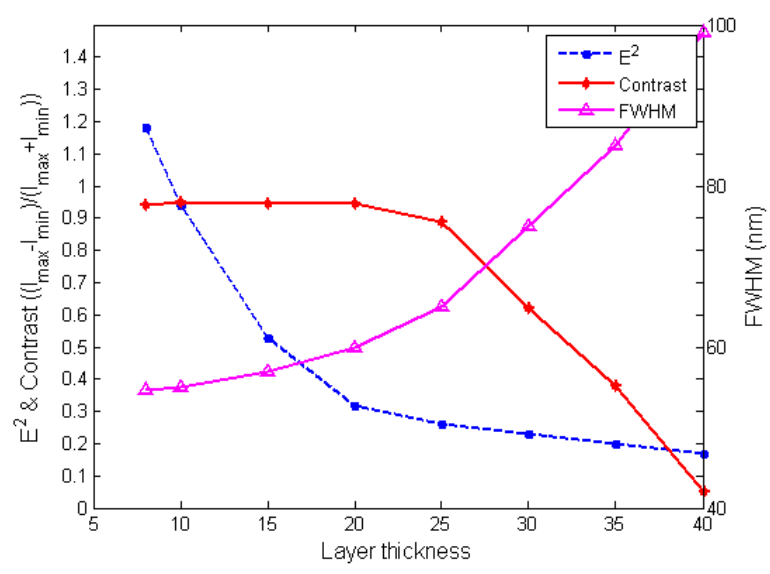

Figure 6. Relation curves between imaging indexes and layer thickness.

Layer number and layer thickness are two main parameters that affect the repeated excitation of surface plasma, as mentioned in Ref.[5,7]. Their research shows that the increase of divergence of the output beam follows the increase of layer number or layer thickness. Our simulation (Fig .5 and Fig .6) agrees with them very well. Judging from the figures, the fewer layer number or layer thickness the better imaging performance.

But layer number or layer thickness changing will unavoidably affect the curvature radius, which plays an important role in imaging performance and scaling. As shown in Fig .7(a).

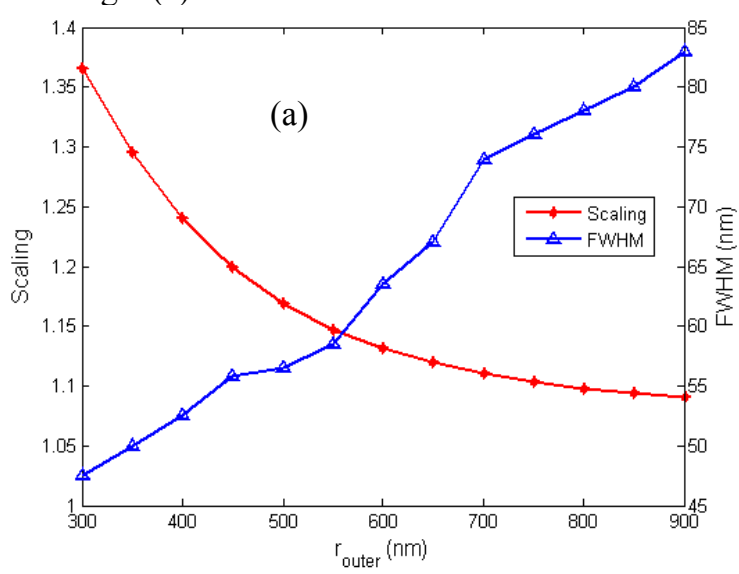

Figure 7. (a) Relation curves between imaging indexes (Scaling, FWHM) and radius 


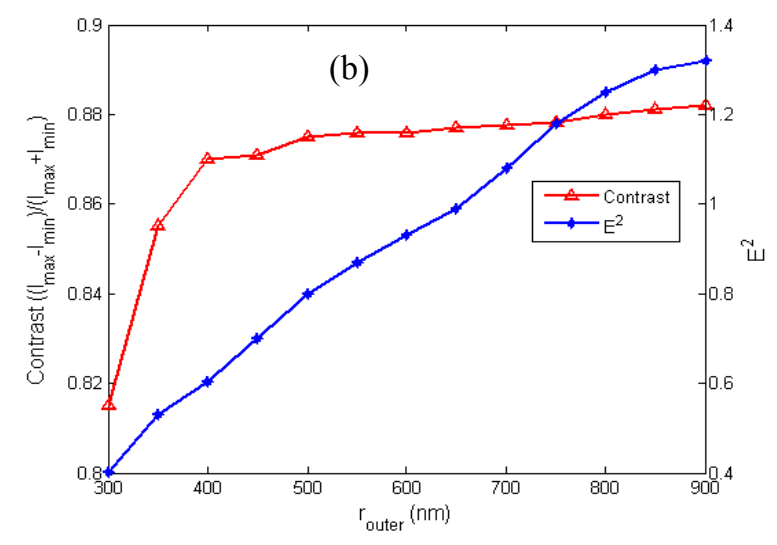

Figure 7. (b) Relation curves between imaging indexes (contrast, Electric field intensity) and radius

From Fig .7(a,b), for one thing, the decrease of radius brings small resolution also leads to the decrease of electric field intensity, which goes against the photoreception of PR. For another, the increase of radius enhances the electric field intensity, but $r_{\text {inner }} / r_{\text {outer }}$ decreases, thus, the scaling that is proportional to $r_{\text {inner }} / r_{\text {outer }}$ decreases too, so the optimal size of the curvature radius depends on the actual demand.

\section{The impact of PRL and $P R$}

PRL helps to improve imaging performance by enhancing both resolution and images intensity[8,12]. The feature reflecting evanescent wave shared by common metals, but their reflecting abilities are different due to different metals. We simulate the imaging performance with different materials $\mathrm{Al}, \mathrm{Au}, \mathrm{Ag}, \mathrm{Cu}, \mathrm{Fe}$ and $\mathrm{Ni}$, then find that $\mathrm{Cu}, \mathrm{Ag}, \mathrm{Ni}$ are relatively well. Fig .8(a-c) shows the electric field intensity distribution of the three metals and $\mathrm{Ag}$ is the best. Considering the cost, $\mathrm{Cu}$ can be the substitute sometimes.

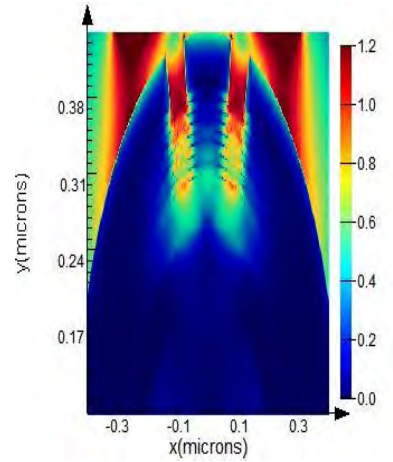

(a) $\mathrm{Ni}$

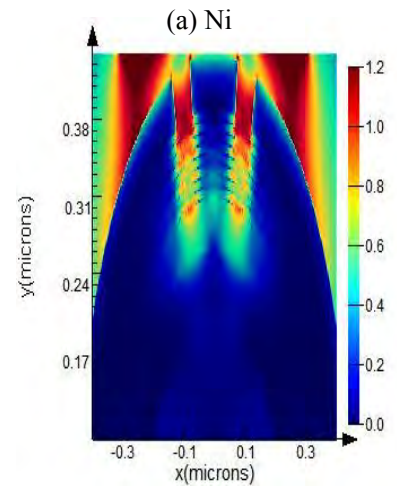

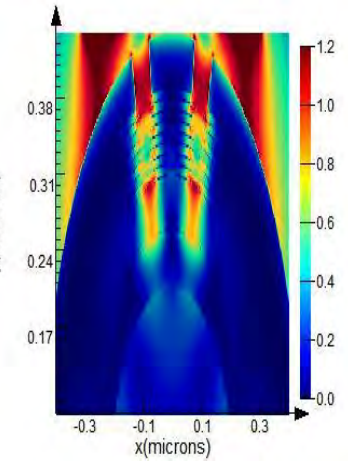

(b) Ag
Figure.8 (a-c) The electric field intensity distribution of three different PRL matels.
Given the PRL Ag, the influence of its thickness should also be optimized. Considering from FWHM shown in Fig .9, the thickness should be greater than 40nm or less than $18 \mathrm{~nm}$.

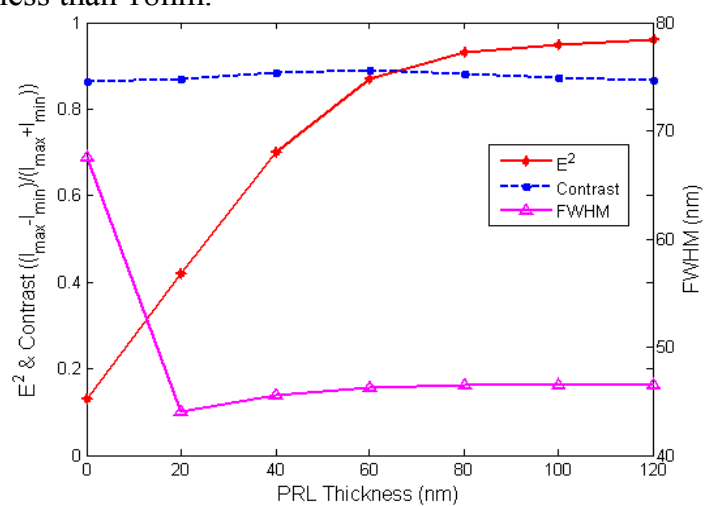

Figure 9. relation curves between imaging indexes and PRL thickness.

But the Contrast begins to fall when PRL thickness is greater than $60 \mathrm{~nm}$. Meanwhile, the electric field intensity is 0.68 when PRL thickness is fixed at $40 \mathrm{~nm}$ and continues to increase afterwards. Directly, the range of PRL thickness should be located at $40 \mathrm{~nm}$ to $80 \mathrm{~nm}$, we choose $60 \mathrm{~nm}$. Of course, the result will be different if the PRL material is $\mathrm{Cu}$ or other metals.

PR is direct imaging part of the hyperlens, naturally, changing of its thickness will affect the indexes of imaging performance. The FWHM presented in Fig .10 is proportional to PR thickness, so the thinner thickness the better imaging performance.

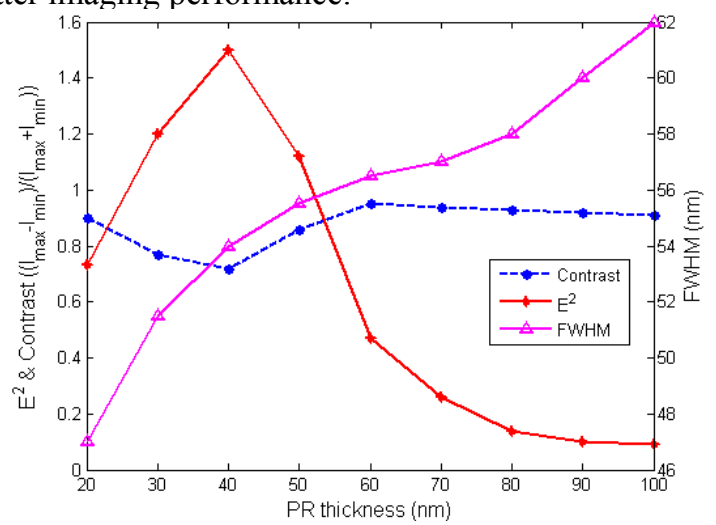

Figure 10. Relation curves between imaging indexes and PR thickness.

But thinner thickness of PR corresponds to weaker electric field intensity. The Contrast keeps above 0.6, while the electric field intensity will experience a period of weak electric field intensity before $20 \mathrm{~nm}$, and decreases rapidly to 0.4 beyond $40 \mathrm{~nm}$, which is harmful to photoreception of PR. The decrease of PR thickness widens the distance between multilayer structure and PRL, which will weaken the transformation from high frequency spatial information to Surface plasma mode. From above discussions, it is proper to choose the thickness of PR from $30 \mathrm{~nm}$ to $50 \mathrm{~nm}$.

\section{CONCLUSION}

We simulate and analyze the multiparameters including medium material, geometric parameters, PRL

(c) $\mathrm{Cu}$ 
and PR. $\mathrm{Ag}$ and $\mathrm{Al}_{2} \mathrm{O}_{3}$ is the best metal and dielectric based on the same thickness of the medium and the wavelength $365 \mathrm{~nm}$. To gain smaller resolution, less layer number and thinner layer thickness will be better, but that results in changing of radius, which also affects the imaging performance and scaling. So the optimal size of the curvature radius may fluctuate for different fabrication of hyperlens. According to our work, Ag is the best selection for PRL and $\mathrm{Cu}$ can sometimes be the substitute for decreasing the cost. The optimal PRL thickness varies from 40 to $80 \mathrm{~nm}$ and the optimal range of PR thickness is between $30 \mathrm{~nm}$ and 50nm.

\section{ACKNOWLEDGMENT}

This work was financial supported by the National Basic Research Program (973 Program) (2013CBA01700) and the National Natural Science Foundation of China (60772026).

\section{REFERENCES}

[1] Xiang zhang and Zhaowei liu, "Superlenses to overcome the diffraction limit," [J],Nature Materials, vol.7,2008, 7, 435-441.

[2] J. B. Pendry, D. Schurig and Nitll D. R. S, "Controlling Eleetromagnetic Fields," [J, Sclence, 2006, 312(35), 1780-1781.

[3] M. Yan, W. Yan, and M. Qiu. "Cylindrical superlens by a coordinate transformation," [J], Phys. Rev. B, 2008, 9, 78(12), 113-115.
[4] Guo-wei Ren, Chang-tao Wang, Guang-wei Yi, Xing Tao and Xian-gang Luo, "Subwavelength demagnification imaging and lithography using hyperlens with a plasmonic reflector layer," [J], Plasmonics, 2013,8, 1065-1072.

[5] Wei-ping Wang,Guo-xing Zheng, "The impact of parameters of hyperlens with artificially engineered metamaterial on subdiffraction imaging," [J], Adv. Mat. Re.2012,Vols, 482-484.

[6] Dong-dong Li, Chang-chun Yan and Dao-hua Zhang, "Geometric Parameter Optimization of Far-Field Hyperlens for Super Resolution," [J], Photonics and Optoelectronic (SOPO), 2010 Symposium on, vol. no. pp.1,3, 19-21.

[7] Ji-Gang H, Cao yong and Wang Pei, "Parameter study on farfield sub-diffraction-limited hyperlens," [J], Chin. Phys. 2008,vol.25, 4151-4153.

[8] LI Heng-yi, WANG Chang-tao and LUO Xian-gang. "Planar Hyper Lens with Demagniifcation for Nanolithography." [J], Opto-Electronic Engineering, 2011,38(5),35-39,45.

[9] Wen-jing guo. "The imaging performance of Curved multilayer super-lens," [D], shenzhen university, 2014.

[10] Jacob Z, AlekseyevLV and Narimanov E. "Semiclassical theory of the hypedens," [J], Opt. Soc. Am. A.2007 Oct;24(10), 54-61.

[11] Z.Liu, H.Lee, Y. Xiong, C. Sun,and X. Zhang, "Far-field optical hyperlens magnifying sub-diffractionlimited objects," $[\mathrm{J}]$, Science. March 2007, Vol. 315, pp, 1686-1687,

[12] John E. Foulkes and Richard J. Blaikie, "Performance enhancements to absorbance-modulation optical lithography. II Plasmonic superlenses," [J], Opt. Soc. Am. A. 28. 2011, 22182225 . 